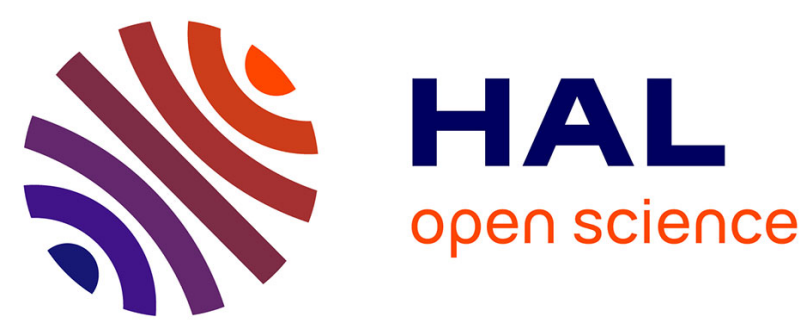

\title{
Extraction et utilisation des granites en Armorique romaine: l'exemple de la carrière de Locuon en Ploërdut (Morbihan)
}

Yvan Maligorne, Jean-Yves Éveillard, Louis Chauris

\section{- To cite this version:}

Yvan Maligorne, Jean-Yves Éveillard, Louis Chauris. Extraction et utilisation des granites en Armorique romaine: l'exemple de la carrière de Locuon en Ploërdut (Morbihan). Gallia - Archéologie de la France antique, 2002, Carrières antiques de la Gaule, 59, pp.133-143. 10.3406/galia.2002.3101 . hal-01912501

\section{HAL Id: hal-01912501 \\ https://hal.science/hal-01912501}

Submitted on 20 Jan 2020

HAL is a multi-disciplinary open access archive for the deposit and dissemination of scientific research documents, whether they are published or not. The documents may come from teaching and research institutions in France or abroad, or from public or private research centers.
L'archive ouverte pluridisciplinaire $\mathbf{H A L}$, est destinée au dépôt et à la diffusion de documents scientifiques de niveau recherche, publiés ou non, émanant des établissements d'enseignement et de recherche français ou étrangers, des laboratoires publics ou privés.

\section{(ㅇ)(1) $\$$}

Distributed under a Creative Commons Attribution - NonCommercial - NoDerivatives $\mid 4.0$ 


\title{
EXTRACTION ET UTILISATION
}

\section{DES GRANITES EN ARMORIQUE ROMAINE}

\section{L'exemple de la carrière de Locuon en Ploërdut (Morbihan)}

\author{
Yvan Maligorne, Jean-Yves Éveillard et Louis Chauris
}

Mots-clés. Locuon, Ploërdut, carrière, granite, architechure, Armorique romaine.

Key-words. Locuon, Ploërdul, quarry; granite, architecture, Roman Armorica.

Şésumé. L'utilisation des granites régionaux constitue l'originalité majeure de l'architecture et de la sculpture en Armorique romaine. I) n ne connâ̂t pour le moment dans la région qu'une seule carrière orientée vers l'extraction de blocs de grand appareil. Située à Locuon Ploërdut (Morbihan), elle a largement approvisionné les chantiers de Vorgium/Carhaix, chef-lieu des Osismes, distant de 27 km.

L a pierre est un granite très clair, blanchâtre et relativement tendre, deux facteurs qui ont cerlainement déterminé son choix. Elle était X raite par la méthode des tranchées verticales creusées à l'escoude. L'acheminement vers Vorgium était assuré par la voie VannesLarhaix, sur le passage de laquelle la carrière s'est développée. Une fouille d'étendue limitée, pratiquée durant l'été 2000, a livré

1). mobilier permettant de dater l'exploitation entre la première moitié du Ir s. et la fin du II's.

Abstract. One of the main originalities of the sculpture and architecture of Roman Armorica lies in the exlensive use of regional granites. For the time being, only a single ashlar quarry has been recognized in the area being studied: located at Locuon, Ploërdut (Morbihan), it widely supplied Vorgium/Carhaix, capital of the Osismi, with stone. The stone is a very light coloured granite, whitish and relatively soft; two factors that may have determined its choice. It was quarried with vertical trenches cut with a special quarryman pick, called " escoude" in Southern France. The $27 \mathrm{~km}$ long route towards Vorgium used the Vannes-Carhaix road, in the immediate vicinity of which the quarry developed. Limited excavations that took place during summer 2000 allow the dating of the exploitation between the first half of the $1^{\text {st }}$ century $A D$ and the end of the $2^{\text {nd }}$ century $A D$.

L'extraction et, plus largement, le travail de la pierre n'ont que très superficiellement retenu l'attention des chercheurs qui se sont consacrés à l'Armorique romaine. L'absence, jusqu'à une date récente, d'outils de référence proposant une méthode de prospection et d'analyse des carrières antiques explique pour une large part ce désintérêt. Il a fallu bien des circonstances heureuses, liées à l'histoire très particulière du site, et tout l'enthousiasme de Marcel
Tuarze, chercheur désireux de faire connaître une carrière dont il pressentait l'antiquité, pour que soit enfin identifié un site d'extraction d'époque romaine en Armorique.

Avant d'aborder l'étude de la carrière de granite de Locuon, il nous a semblé indispensable de donner un aperçu de la géologie régionale, puis de nous pencher brièvement sur la place qu'occupait le granite dans le travail de la pierre à l'époque romaine. 


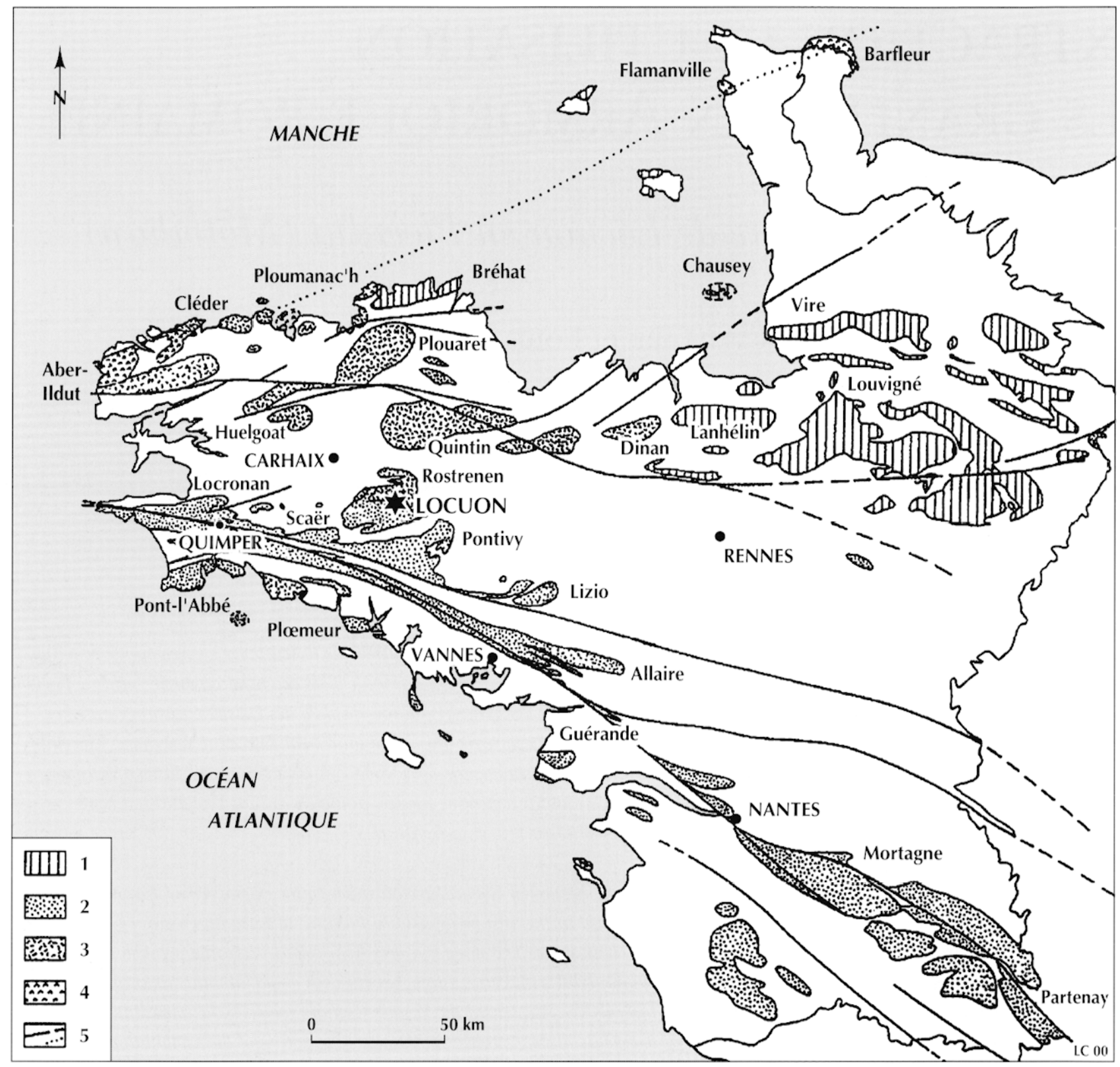

Fig. 101 - Esquisse très simplifiée des intrusions granitiques du Massif armoricain : 1, granites cadomiens (ì biotite) ; 2-4, granites hercyniens (2, ceinture leucogranitique avec muscovite ; 3, ceinture des granites à biotite; 4, ceinture des granites rouges) ; 5 , failles majeures (I)AO I. (Chauris et (s. Couix, unix. de Brest).

\section{PRÉSEN'IA'TION SOMMAIRE \\ DE LA GÉOLOGIE \\ DE LA PÉNINSULE ARMORICAINE}

Le Massif armoricain se caractérise par une extrême diversité pétrographique: citons, dans une énumération qui n'est pas exhaustive, les granites, les gneiss, les schistes, les quartzites et même les calcaires, sans oublier les multiples roches filoniennes, telles que les dolérites ou les kersantites. Si un certain nombre de ces roches ont été exploitées à l'époque romaine pour obtenir des moellons de petit appareil, la taille des blocs de grand appareil, et principalement du décor architectonique, s'est heurtée à deux contraintes majeures: l'omniprésence des massifs granitiques de faciès variés, et la rareté, principalement dans la partie occidentale de la contrée, des roches calcaires, a fortiori des marbres. 
Les venues granitiques appartiennent aux deux orogénèses majeures qui ont marqué la longue histoire de l'Armorique (fig. 101) : a, la chaîne cadomienne, à la fin des temps briovériens, avec la mise en place du puissant batholite mancellien (Louvigné-du-Désert, Vire, Lanhélin) et du batholite côtier trégorrois (Bréhat...), où dominent largement les granites à grain fin ou moyen à biotite (mica noir) ; b, la chaîne hercynienne, à la fin de l'ère primaire, avec l'intrusion de trois ceintures batholitiques, convergeant vers l'ouest, caractérisées respectivement: au sud, par des leucogranites souvent riches en muscovite (mica blanc), s'étirant sans interruption de la pointe du Raz au seuil du Poitou; au centre, par des granites fréquemment à gros grain, plus rarement à grain lin, soit à biotite seule, soit à deux micas, dessinant une succession de plutons séparés, de Saint-Renan à Dinan; au nord enfin, par des pointements granitiques isolés, connus sous le nom de " traînée moniliforme des granites rouges ", comparés ainsi aux perles d'un collier.

Les dépôts calcaires armoricains se rapportent à différentes étapes du Primaire: Cambrien, Ordovicien, Dévonien et Carbonifère. Ce sont des roches le plus souvent de teinte assez sombre, fréquemment d'extension réduite, dures, plissées et faillées, ce qui limite leur emploi ; mais ces matériaux ont pu fournir un précieux mortier. Les dépôts d'âge tertiaire - clairs et tendres, grossiers et coquilliers - sont encore beaucoup plus restreints (Le Quiou...).

\section{UTILISATION DU GRANITE DANS LA PÉNINSULE ARMORICAINE À L'ÉPOQUE ROMAINE}

La pénurie en roches calcaires de la péninsule armoricaine a certes été atténuée par l'importation de matériaux extraits des bassins secondaires limitrophes (tendre tuffeau de l'Anjou, calcaire plus résistant des Charentes) et transportés facilement par voie d'eau. Ces pierres étaient réservées à trois usages : le décor architectonique, la statuaire, le décor pariétal.

Il est difficile d'évaluer la part des différents matériaux dans le décor architectonique: les données sont manifestement faussées par la fréquente destruction des blocs de calcaire par les chaufourniers. Le décor du forum de Vannes, entièrement sculpté dans le tuffeau, en fournit la parfaite illustration : les fouilles n'ont mis au jour que des fragments très réduits, dont plusieurs portent des traces de sciage témoignant de leur débitage.

Il est malgré tout possible de proposer un tableau d'ensemble. Nantes et Vannes semblent avoir massivement fait appel à des calcaires acheminés depuis le Val de Loire et les Charentes. La place du granite y est très modeste, voire marginale, et les deux agglomérations ont manifestement profité de leur situation portuaire pour importer un matériau plus facile à travailler que le granite local.

À Carhaix et Rennes, la présence du calcaire est plus sporadique: dans la capitale des Osismes, les plus riches demeures faisaient appel au tuffeau, ainsi, semble-t-il, que certains monuments funéraires; les monuments publics, en revanche, dont le décor mobilisait des volumes beaucoup plus importants, paraissent n'avoir utilisé que le granite régional. Dans le cheflieu des Riedones, l'enceinte tardive a assuré la conservation de nombreux éléments d'architecture; on est en droit de penser que sa composition reflète assez fidèlement les proportions des différents matériaux mis en œuvre sous le Haut-Empire. Ici encore, le tuffeau du Val de Loire, s'il n'est pas absent, s'est largement vu préférer le granite.

Corseul, enfin, présente une situation plus tranchée : le calcaire comme le marbre y sont seulement représentés par des éléments de placage (petites corniches et architraves, dalles, revêtements pariétaux...) et le décor architectonique recourt uniquement à un granite extrait dans le massif de Languédias, à une dizaine de kilomètres au sud de l'agglomération.

Au total, et malgré les réserves exprimées plus haut sur la représentativité des éléments conservés, nous pouvons avancer que le granite régional était le matériau le plus couramment utilisé dans les cités des Osismes, des Coriosolites et des Riedones. Cela n'est évidemment pas sans conséquence sur la nature de la production : même si le choix des bâtisseurs s'est en priorité porté sur des granites très fins et très homogènes, les éléments d'architecture sont en général très sobres; les chapiteaux toscans dominent très largement, ainsi que des corniches ou architraves au profil très simplifié. On n'en déduira pas que le matériau a opposé des contraintes insurmontables à l'élaboration de modénatures complexes ou de motifs finement ouvragés - l'hypothèse d'un " déterminisme géologique " est infirmée par quelques réalisations 
très honorables ${ }^{85}$ - mais simplement que le granite imposait une taille plus longue et, partant, plus coûteuse, ce qui a manifestement découragé les commanditaires.

La sculpture figurative pose évidemment des problèmes fort différents : les volumes de pierre sont infiniment moindres, et l'acheminement de matière première, voire d'œuvres achevées, est plus facilement envisageable. Certes, le recours à l'importation est bien attesté (Éveillard, 1998, p. 78-80), mais l'on dispose maintenant de multiples preuves que les artisans régionaux n'ont pas dédaigné le granite, réussissant même à tirer de certains faciès ingrats des œuvres remarquables. On retiendra par exemple la partie inférieure d'un tronc masculin dénudé, découverte à Corseul en 1991 et façonnée dans le granite fin de Languédias; le respect des proportions, l'exactitude du modelé révèlent un artiste maîtrisant parfaitement le matériau. Un savoirfaire équivalent transparaît encore, malgré les mutilations et l'altération de la pierre, dans trois groupes complexes de grandes dimensions - le plus grand atteignait $2 \mathrm{~m}$-, découverts dans un rayon de $10 \mathrm{~km}$ autour de Quimper; il s'agit de trois cavaliers à l'anguipède. Dans cette partie de la péninsule, où abondent les leucogranites, les sculpteurs ont à chaque fois choisi une pierre de qualité, extraite non loin du lieu d'érection des monuments. Mais le plus remarquable nous semble être une tête isolée, probablement de Jupiter, provenant de Plogonnec, à $12 \mathrm{~km}$ au nord-ouest de Quimper. Malgré l'ingratitude du matériau, un granite dur et à grain plus grossier, l'artiste a su conférer au visage un modelé nuancé et une expression impérieuse, qui rivalisent avec des ouvres sculptées ailleurs dans le calcaire ou dans le marbre.

Ce bref aperçu montre que les massifs granitiques régionaux étaient à l'évidence largement exploités. Le fait n'était pas nouveau : le Néolithique, avec ses innombrables mégalithes, et la charnière entre le premier et second âge du Fer, avec des centaines de stèles funéraires, avaient déjà vu se développer une intense activité d'extraction et de taille de la pierre. Mais avec la conquête romaine, les bâtisseurs et tailleurs de pierre réussissent à s'affranchir des contraintes du substrat géologique immédiat, n'hésitant pas à transporter la pierre sur des dizaines de kilomètres.

85. Outre les blocs de Carhaix présentés infra, voir un chapiteau à feuilles d'acanthe de Tréogat, dans la cité des Osismes (Peuziat, 1999).

\section{EXTRACTION ET PREMIÈRE MISE EN FORME DU GRANITE : UNE ÉTUDE DE CAS}

Extrêmement rares sont les carrières assurément antiques identifiées dans la région. La pauvreté de l'information ne fait en la matière que refléter le manque d'enthousiasme des chercheurs pour des questions qui reçoivent ailleurs - on songe par exemple aux Germanies ou à la Narbonnaise - une attention soutenue. En 1983, P. Galliou pouvait même déplorer qu'aucun site d'extraction d'époque romaine ne fût connu (Galliou, 1983, p. 143-144), et les maigres progrès enregistrés depuis lors sont plus l'effet de hasards heureux que le résultat de recherches systématiques soutenues par des problématiques cohérentes.

Toutes les carrières repérées ne sont d'ailleurs pas utiles à notre propos : ainsi, la petite excavation identifiée par P.-R. Giot sur l'île-Lavret, dans l'archipel de Bréhat (cité des Osismes), n'a-t-elle produit que des moellons de petit appareil utilisés pour la construction de l'habitation voisine. La pierre y était extraite à l'aide de coins, en mettant à profit le système de diaclases qui fracturent la roche (Giot, 1996, p. 17). La production de la carrière de gneiss mise au jour par Y. Ménez, à Plouërsur-Rance (cité des Coriosolites), aux abords immédiats d'un établissement rural, aurait quant à elle été diffusée plus largement et l'activité extractive aurait servi à dégager des surplus, sans être cantonnée à un usage strictement local (Ménez, 1996, p. 88 et 194). Là encore, néanmoins, il s'agissait de façonner des moellons: des coins ou pinces de carriers ont dû suffire à l'extraction d'une roche très fractionnée.

Une seule carrière orientée vers l'extraction de blocs de grand appareil est donc actuellement connue dans les limites du Massif armoricain. Située à Locuon, sur la commune de Ploërdut, à proximité de la frontière présumée entre Osismes et Vénètes, elle a été ouverte pour approvisionner les chantiers de Carhaix (fig. 102).

\section{SITUATION ET CADRE TOPOGRAPHIQUE DE LA CARRIÈRE DE LOCUON}

La carrière de Locuon est située aux confins nord de la commune de Ploërdut (Morbihan). Par rapport à la ville de Vorgium/Carhaix, dont elle a approvisionné majo- 


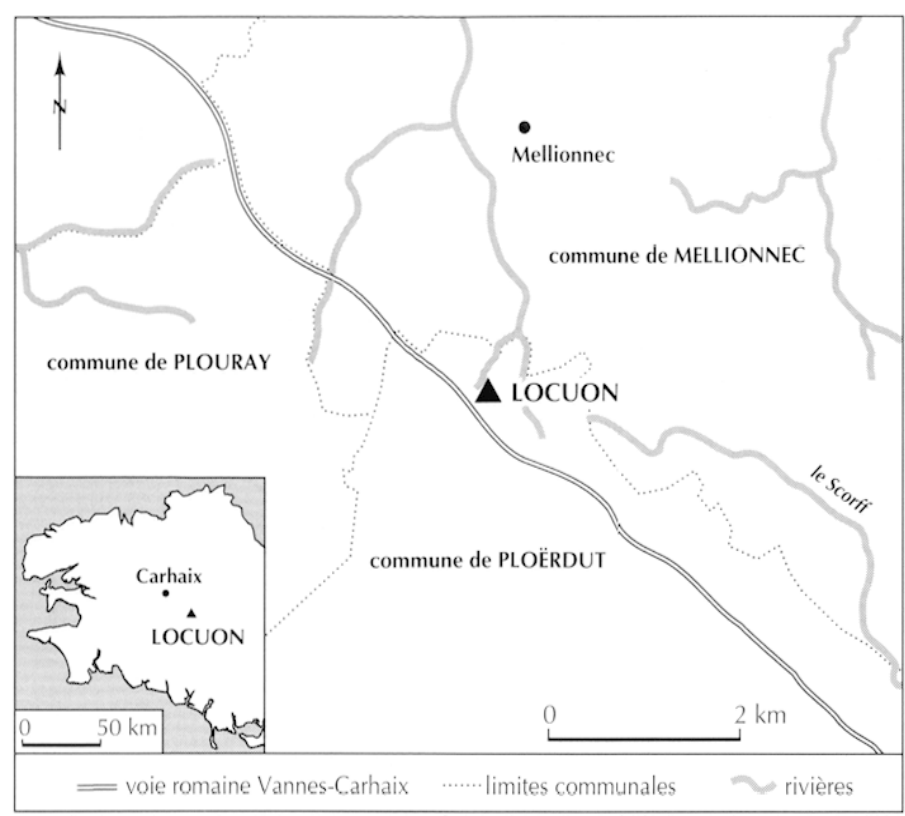

Fig. 102 - Carte de localisation de la carrière de Locuon en Ploërdut, Morbihan (DAO G. Couix, univ. de Brest).

ritairement les chantiers en pierre de grand appareil, elle est éloignée de $27 \mathrm{~km}$ quand on emprunte le tracé de la voie antique Vannes-Carhaix. On peut dire qu'elle se trouve sur le passage même de cet itinéraire, puisqu'une distance de $40 \mathrm{~m}$ seulement l'en sépare. Nul doute que la proximité de cet axe de circulation a constitué un facteur favorable à son développement.

L'éminence sur laquelle s'étend le bourg de Locuon culmine à $262 \mathrm{~m}$; elle a été entaillée par plusieurs carrières (fig. 103) : l'une, située à environ $60 \mathrm{~m}$ au sudest de l'église, a été méthodiquement rebouchée pour être transformée en prairie ; une deuxième, s'étendant à l'ouest de l'église, a laissé place à un jardin d'où n'émerge que le sommet des fronts de taille*.

Une seule carrière est actuellement accessible. Située au nord-est de l'église, dont le chevet n'est qu'à $11 \mathrm{~m}$ du front le plus proche, elle couvre plusieurs hectares, en grande partie envahis par la végétation et les déchets d'extraction. Elle se présente aujourd'hui comme une exploitation en tranchée, établie à flanc de coteau, perpendiculairement aux courbes de niveau ${ }^{86}$; mais cette organisation résulte vraisemblablement d'une reprise de l'extraction à l'époque moderne. Il apparaît en effet que la hauteur de la découverte, pratiquement nulle au

86. Pour les différents types d'extraction à ciel ouvert, voir Bessac, 1986b, p. 169-170, fig. 13.

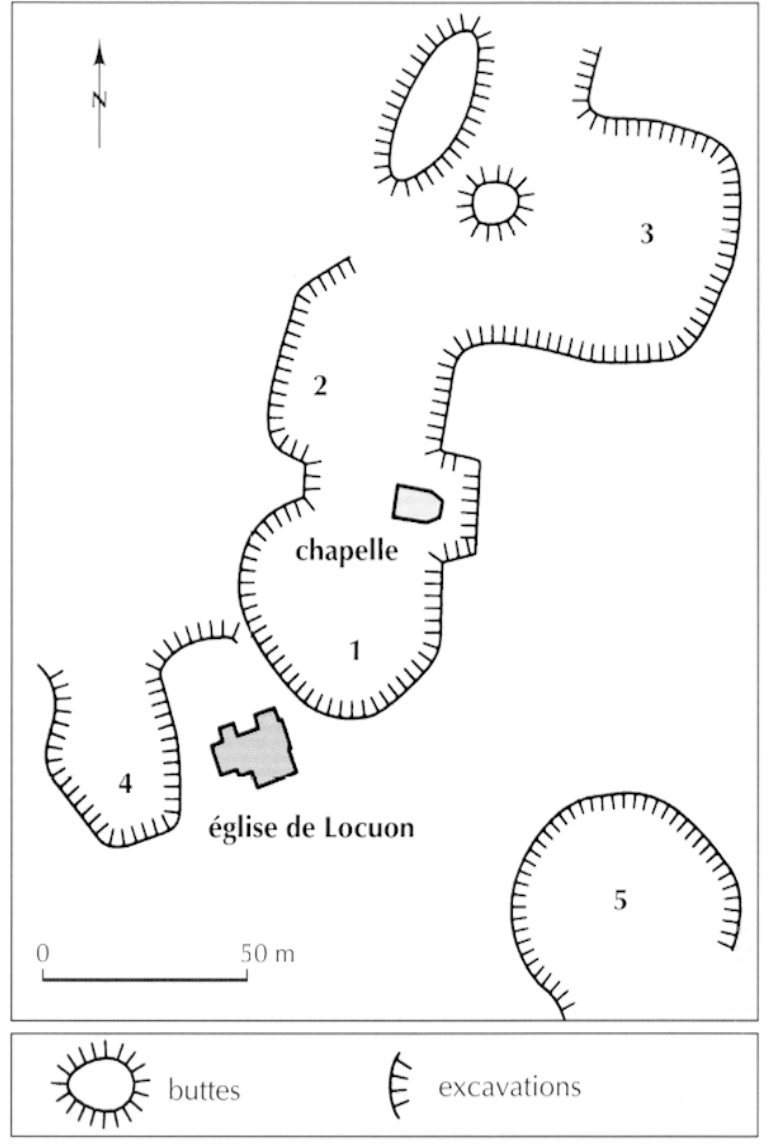

Fig. 103 - Plan schématique des sites d'extraction de la carrière de Locuon en Ploërdut, Morbihan (DAO (;. Couix, univ. de Brest).

sommet du coteau, où le granite affleure en plusieurs points, augmente progressivement vers le nord jusqu'à atteindre $3 \mathrm{~m}$. L'exploitation a donc dû s'enfoncer en fosse dans la partie méridionale de la carrière, celle qui précisément se prête le mieux à l'observation (fig. 104). Ce secteur a fait l'objet à l'époque moderne d'un aménagement monumental qui l'a en partie préservé du comblement : au XVII ${ }^{\mathrm{c}}$ ou au XVIII ${ }^{\mathrm{c}}$ s., furent construits une chapelle et un escalier. La chapelle, érigée à quelques mètres d'un front de taille, fournit un terminus ante quem important pour la datation de l'arrêt de l'extraction, d'autant que, l'édifice étant établi sur une importante couche de déblais, l'exploitation devait avoir cessé depuis de nombreuses années au moment de sa construction (fig. 105).

Deux fronts de taille* sont bien dégagés : l'un au nord, orienté est-ouest et dont la longueur développée ne dépasse pas $10 \mathrm{~m}$; l'autre, à l'est, visible sur près de $60 \mathrm{~m}$ et sur une hauteur qui atteint jusqu'à $8,50 \mathrm{~m}$ (fig. 106). C'est sur ces deux parois qu'a porté l'essentiel de nos 


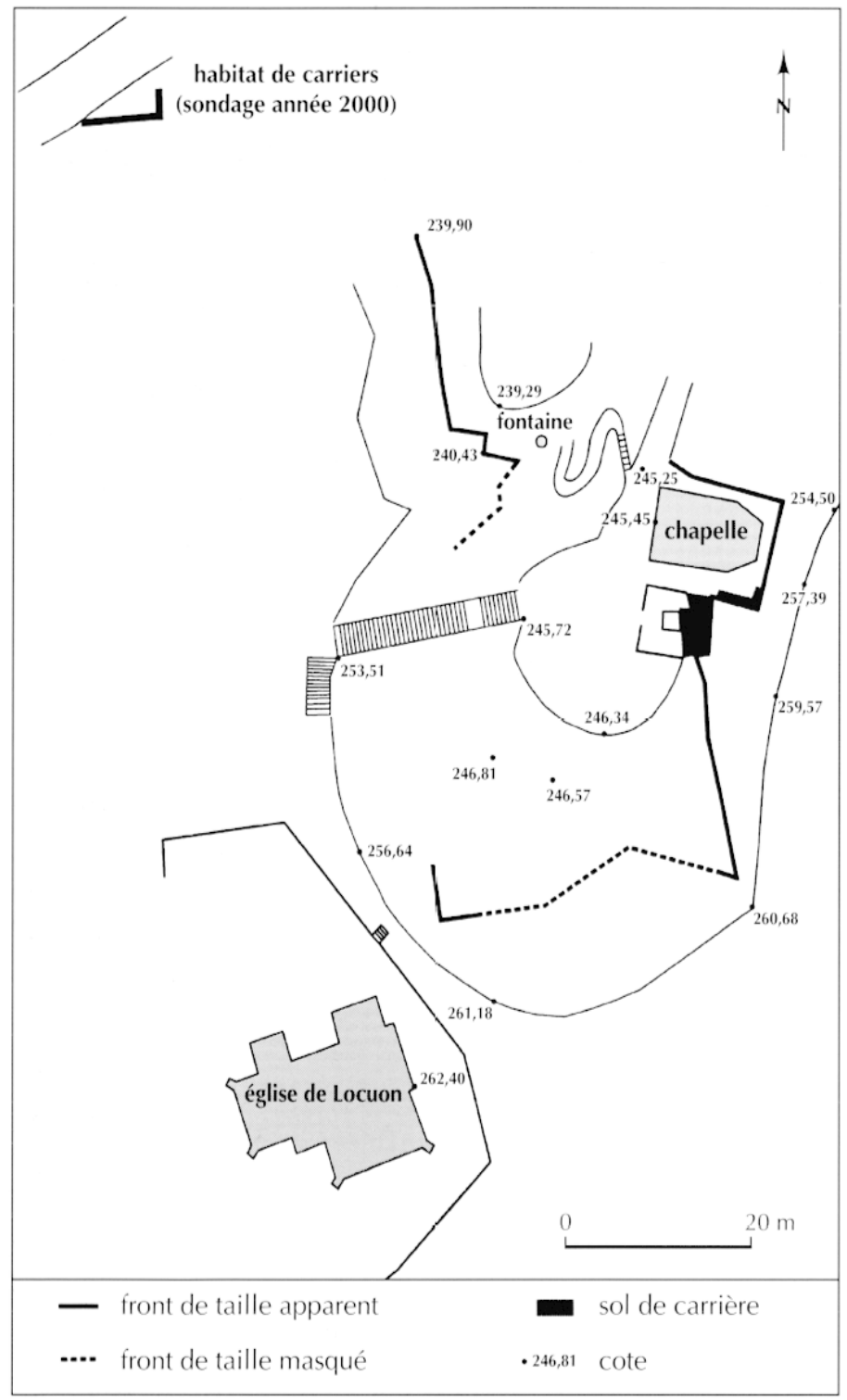

Fig. 104 - Plan du secteur méridional exploité à l'époque romaine (I)AO (;. Couix, unit. de Brest).

observations, auxquelles les récents travaux de J.-C. Bessac sur les carrières du massif du Bois des Lens, dans la région nîmoise, ont fourni un cadre méthodologique précis (Bessac, 1996, p. 81-111 et supra, p. 29-51).

\section{ÉTUDE GÉOLOGIQUE DE LA CARRIÈRE DE LOCUON}

Le granite extrait dans la carrière de Locuon représente l'un des faciès du massif leucogranitique de Langonnet, intrusif dans le pluton de Rostrenen.

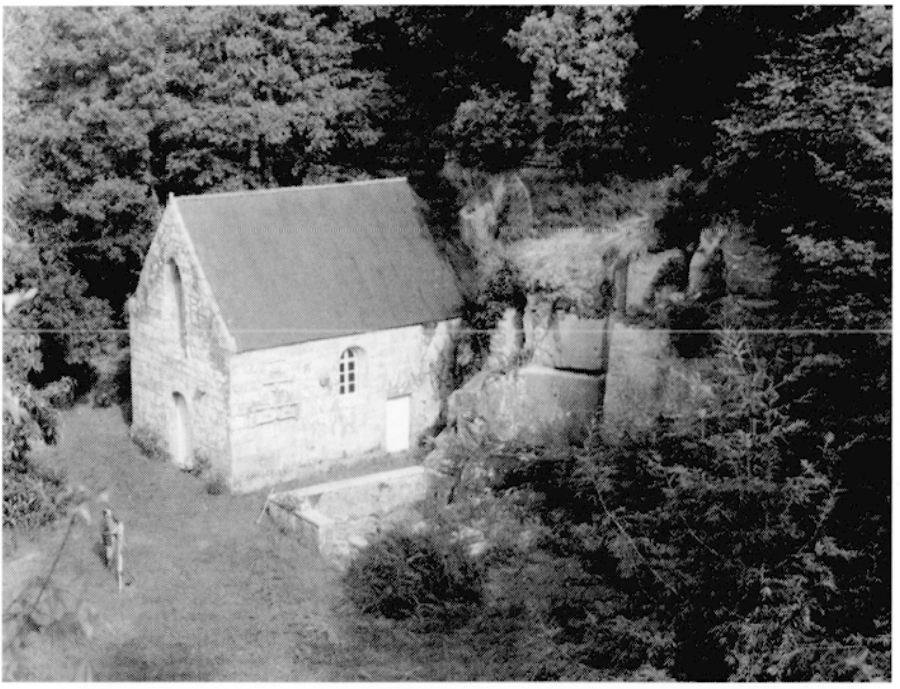

Fig. 105 - Le secteur méridional : vue depuis le sud-ouest (photo J.-Y. Éveillard, univ. de Brest).

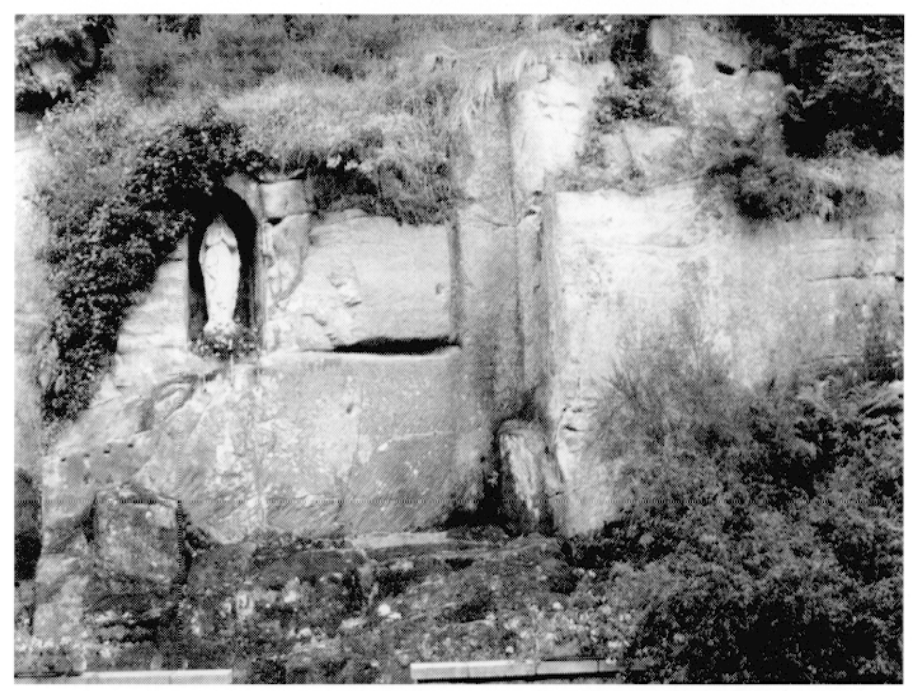

Fig. 106 - Le fronı de laille oriental avec aménagements modernes (photo J.-Y. Everillard, unit. de Brest).

La pierre de Locuon est particulièrement claire, blanchâtre, à l'aspect quasi neigeux ; par altération, elle peut prendre une nuance jaunâtre. La granulométrie est fine, de l'ordre du millimètre. Le feldspath, constituant essentiel, est terne; le quartz gris, à éclat brillant; la muscovite (mica blanc) est assez sporadique. Aucune orientation préférentielle n'est mise en évidence. À la différence d'autres granites à grain fin du massif de Langonnet, qualifiés par les carriers, dans leur langage imagé, de " raides ", la pierre de Locuon est reconnue comme "molle ». J.-C. Bessac, qui s'est livré à des essais de taille, la compare à certains grès et aux granites de la 
région de Vigo, dans la péninsule Ibérique ; il la situe aux indices 7-8 de la norme AFNOR, c'est-à-dire à la frontière des catégories fermes et dures (communication personnelle). Tendre, relativement facile à travailler, elle résiste toutefois bien à l'érosion après sa mise en oeuvre. À l'évidence, la faible dureté de ce granite, asse\% exceptionnelle, a dû jouer un rôle majeur dans son utilisation intensive à l'époque romaine : toute proportion gardée, cette caractéristique physique du matériau le rapprochait un peu, pour l'exploitant, des roches calcareuses.

Cette propriété pétrographique favorable - nécessaire mais encore insuffisante - était heureusement complétée par des critères structuraux également propices à l'exploitation. En premier lieu, une disposition en bancs généralement peu inclinés, évoquant encore, pour les carriers, les roches sédimentaires, offrant ainsi des plans naturels de séparation au sein de la masse rocheuse. Mieux, en profondeur, lesdits bancs acquièrent rapidement des épaisseurs convenables pour l'obtention des blocs de grand appareil, atteignant et même dépassant largement $1 \mathrm{~m}$, voire même parfois 1,50 m. Par ailleurs, l'espacement des diaclases subverticales qui recoupent le granite selon des directions à peu près perpendiculaires conditionne aussi le volume des blocs à extraire.

Ainsi, près de la fontaine, il est respectivement de $1,65 \mathrm{~m}$ et de $0,50 \mathrm{~m}$, ce qui, avec une épaisseur des bancs, à la partie inférieure du front de taille, de l'ordre de $1,10 \mathrm{~m}$ - et compte tenu de la perte de matière due au mode d'extraction -, permet d'obtenir des éléments de $1 / 2 \mathrm{~m}^{3}$ environ, soit, avec une densité du granite de 2,6, de 1,3 t. En d'autres points de l'excavation (près de la chapelle), les espacements deviennent nettement plus considérables, respectivement de l'ordre de $1,10 \mathrm{~m}$ et de 1,30 m, ce qui conduit ici à l'obtention de blocs atteignant le mètre cube $(2,6 \mathrm{t})$.

Il faut se garder toutefois de se représenter la carrière de Locuon comme une masse homogène de granite, entièrement utilisable pour la pierre de taille. Comme partout, apparaissent ici aussi des zones failleuses où la densité du diaclasage est telle que la fourniture de gros blocs est impossible. Ainsi, sur le flanc occidental de la carrière, au nord-ouest de la fontaine, l'écartement des diaclases n'est que de $0,13 \mathrm{~m}$ à $0,25 \mathrm{~m}$. Toutefois, au vu des fronts de taille encore observables, de telles zones de fractures paraissent rares, ce qui augmente encore considérablement l'intérêt du site.

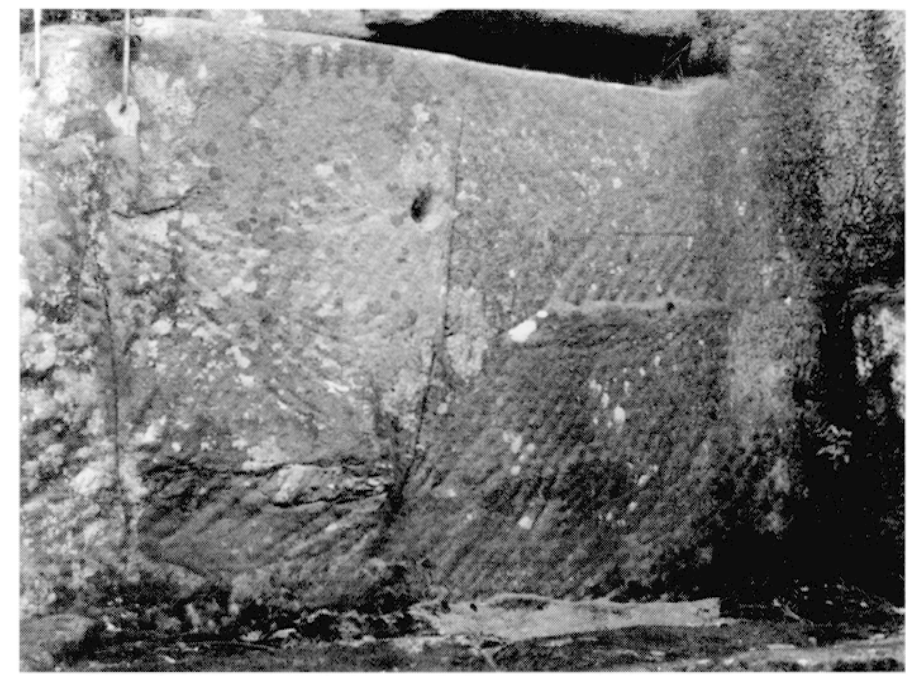

Fig. 107 - Traces d'escoude sur le front de taille oriental (photo J.-Y. Liveillard, univ. de Brest).

Ainsi, à Locuon, facteurs pétrographiques et structuraux se sont conjugués pour offrir, avec abondance, et des conditions d'extraction relativement faciles, un beau matériau homogène, apte au façonnement du grand appareil, exigé par les constructions monumentales de Vorgium/Carhaix. Mieux, la faible dureté du granite armoricain en ce point a permis d'utiliser à l'extrême occident de la Gaule des méthodes d'exploitation comparables à celles pratiquées par les Gallo-Romains dans les calcaires des régions méditerranéennes.

\section{MÉTHODES D'EXTRACTION ET MÉTROLOGIE DES BLOCS EXTRAITS}

Les fronts de la carrière* de Locuon sont parcourus de deux types de traces : d'une part, de longues séries de sillons parallèles et légèrement curvilignes, consécutives à l'extraction proprement dite ; d'autre part, des sillons plus courts et désordonnés, résultant d'une rectification des parois au pic (fig. 107).

Le principe général de l'extraction est bien connu: des tranchées verticales - les traces ${ }^{*}$, ou havages* - étaient creusées autour du volume de pierre qui devait être extrait, puis le bloc était arraché au substrat à l'aide de coins fichés dans une série d'emboîtures ${ }^{*}$ horizontales.

Les tranchées ont ici été ménagées à l'aide d'une escoude $^{*}$ à pointe pyramidale unique. Elles étaient creusées en pratiquant plusieurs passes" frontales; en la matière, aucune constante ne se dégage des traces 
conservées et leur nombre varie de deux à cinq. La largeur des tranchées et, partant, le nombre de passes, étaient en fait directement fonction de la hauteur des blocs désirés. On relève cependant une tendance très nette au rétrécissement des tranchées dans leur partie inférieure; la pratique, qui trouve son origine dans un souci de gain de temps, est simplement limitée par la nécessité dans laquelle se trouve le carrier de placer un pied au fond de la tranchée.

La mesure de l'espacement des sillons visibles sur les parois permet de mesurer, au moins en termes relatifs, l'efficacité de la progression des carriers. Les données recueillies en divers points montrent que l'écart s'établissait généralement autour de $35-40 \mathrm{~mm}$, ce dont on peut déduire que la main-d'œurre possédait un niveau de compétence très homogène.

Nous sommes beaucoup moins bien renseignés sur la dernière phase de l'extraction, qui fait intervenir les coins. Cinq empreintes d'emboîtures sont visibles près du front oriental, mais, trop érodées, elles ne se prêtent pas à des observations précises. Dans le même secteur sont en revanche conservées quatre emboîtures* complètes, donc non utilisées. De forme trapézoïdale, elles sont creusées indépendamment les unes des autres, sans être reliées par une saignée* continue ; elles présentent une ouverture en amande, caractéristique de l'emploi du pic (Bessac, 1996, p. 238). La position de ces emboîtures* sur le front de taille ${ }^{*}$ pose un problème: elles ne s'intègrent pas au canevas d'extraction défini par les blocs extraits, et, surtout, aucune tranchée ${ }^{*}$ verticale ne leur correspond. Il semble en fait que ces cavités doivent être rattachées à une phase ultérieure de l'exploitation, sans doute moderne, et qu'elles aient été pratiquées pour une extraction ne faisant intervenir que les coins, voire pour régulariser un sol de carrière très perturbé à cet endroit.

On le voit, de très nombreuses incertitudes pèsent encore sur les techniques mises en œuvre à Locuon, qui ne pourront être levées que par un dégagement des sols de carrière.

$\grave{A}$ en juger par les empreintes des blocs extraits, la carrière était essentiellement consacrée à la production de blocs de grand appareil. Le fait que les sols de carrière soient largement masqués empêche évidemment le plus souvent de calculer la largeur des blocs, mais leur longueur est matérialisée par les fonds de tranchées* qui subsistent sur les parois, et leur hauteur par de légers ressauts horizontaux. Les hauteurs mesurées ${ }^{87}$ varient de 50 à $118 \mathrm{~cm}$, les longueurs, de 150 à $213 \mathrm{~cm}$. Il est d'ailleurs certain que des blocs plus longs ont été arrachés à la carrière: c'est ce que prouve, avec ses $242 \mathrm{~cm}$ de longueur, l'architrave conservée à Landeleau (cf. infra). La grande variété des dimensions des blocs extraits indique que la production de la carrière n'était pas soumise à des normes préétablies, et que les carriers répondaient à des commandes précises.

Si les bancs les moins épais du massif ont pu être mis à contribution pour des bases ou chapiteaux de petit module, ou encore pour des autels (cf. infra), il est certain que les couches superficielles très fractionnées ne présentaient aucun intérêt pour les carriers : la ville de Carhaix faisait en effet appel, pour ses moellons de petit appareil, à un grès feldspathique strictement local (Éveillard, Maligorne, 2000, p. 63).

\section{LA TAILLE DES BLOCS DANS LA CARRIÈRE}

La carrière de Locuon était aussi le lieu d'un dégrossissage poussé des blocs ; effectué non loin des fronts de taille, il avait évidemment pour fonction première de réduire le poids des blocs en vue du transport. En 1973, lors du percement d'un chemin, quatre blocs inachevés ont ainsi été mis au jour (fig. 108). Le moins significatif, puisque sa destination finale ne peut être déterminée, est un bloc parallélépipédique dont l'une des faces est brute d'extraction, les autres portant des traces de pic ${ }^{*}$ et de broche ${ }^{*}$. Le deuxième est un tambour sur lequel peuvent être observées deux phases du dégrossissage : l'une des sections est grossièrement traitée au pic, l'autre, s'approchant au plus près du volume définitif, est dressée à la broche. Le troisième bloc se présente sous la forme d'un volume cylindrique muni à l'une de ses extrémités d'un épais disque saillant; il s'agit sans doute d'une base de colonne en cours d'épannelage. Enfin, il faut signaler la découverte d'un bloc de chaperon presque entièrement achevé.

Ces divers éléments montrent que l'ébauche en carrière pouvait être poussée jusqu'à un stade très

87. La hauteur de $118 \mathrm{~cm}$ se place aux limites des normes de l'extraction antique. Il faut en outre noter que cette dimension correspond à la hauteur " utile " du bloc; le sol de carrière tómoigne en effet d'un défaut d'extraction au moment de l'arrachement du bloc par les coins et la profondeur effective des tranchées s'établit à $135 \mathrm{~cm}$. 


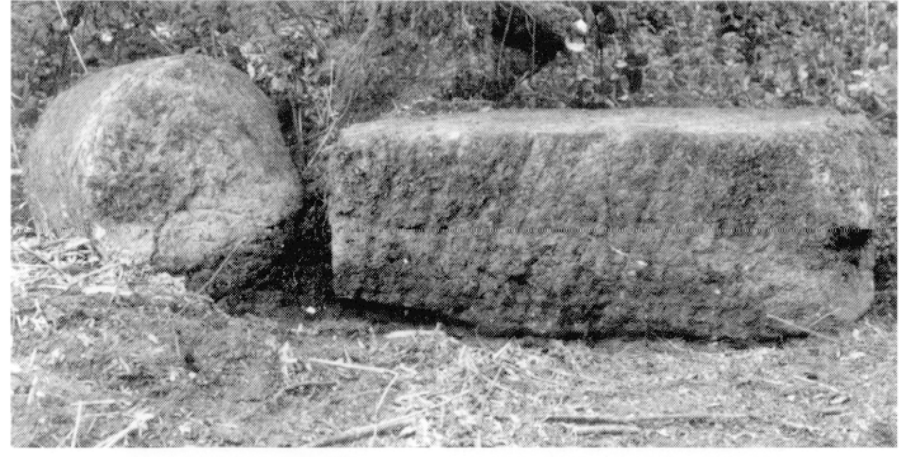

Fig. 108 - Deux des blocs inachevés découverts dans la carrière de Locuon (photo J.-Y. Eveillard, univ. de Brest).

avancé. Ils confirment par là même que les carriers avaient une connaissance précise de la fonction des blocs qu'ils extrayaient.

En marge de ce qui constituait manifestement leur activité principale, à savoir la production de blocs de grand appareil, les carriers se sont livrés à la taille sommaire de petits autels, dont on ne sait pas s'ils devaient être vendus aux populations environnantes ou s'ils étaient destinés aux carriers eux-mêmes. Deux petits monuments sont exposés dans l'église de Locuon : l'un, proche des modèles classiques, est haut de $70 \mathrm{~cm}$; il a conservé son corps parallélépipédique et son couronnement, composé d'une doucine encadrée par deux listels et surmontée par un bandeau. L'autre est plus fruste : haut de $65 \mathrm{~cm}$, il offre un corps tronconique et désaxé, encadré par une base et un couronnement réduits à l'état de bourrelets informes. Ces activités, qui n'ont pu porter que sur des volumes insignifiants au regard de la production globale de la carrière, étaient prolongées par la fabrication d'objets domestiques, comme des meules, dont un exemplaire est conservé dans le bourg.

\section{DATATION DE L'EXPLOITATION}

Les éléments de comparaison font défaut pour analyser dans le détail les méthodes d'extraction en vigueur dans la carrière osisme. Il n'est pour s'en convaincre que d'examiner le problème particulier de la partie active de l'escoude*. L'outil d'extraction utilisé sous le Haut-Empire se caractérisait par la présence, à chacune de ses extrémités, de deux dents. Ce constat, dont la valeur chronologique est établie pour la
Narbonnaise et le Proche-Orient (Bessac, 1993a, p. 154), a pu être étendu à l'Aquitaine sur la foi d'observations plus ponctuelles (Bocquet, Valat, 1995, p. 51) ${ }^{88}$.

L'escoude à pointe pyramidale unique rencontrée à I ocuon correspond quant à elle, dans la typologie établie par J.-C. Bessac, à la forme E, laquelle était employée dans le Bois des Lens du Bas-Empire au haut Moyen Âge (Bessac, 1996, p. 208) ; on retrouve les impacts d'un outil similaire dans les carrières de Saint-Savinien, en Charente-Maritime, au XII" s. (Bocquet, Valat, 1995, p. 51). Or, l'examen pétrographique des monuments de la région de Locuon montre que l'affleurement n'a connu que deux phases d'exploitation: l'une sous le Haut-Empire, l'autre à l'époque moderne, la reprise de l'extraction devant être située à la fin du $\mathrm{XV}^{\mathrm{c}}$ ou au début du XVI ${ }^{e} .{ }^{89}$. Il apparaît ainsi que les données recueillies sur le pourtour méditerranéen, essentiellement dans des carrières de calcaire, ne peuvent être transposées mécaniquement à un autre contexte géographique et appliquées au granite. Il est fort probable que la nature abrasive du matériau a influé sur la morphologie de la pointe de l'outil.

Faute de données intrinsèques, on aurait aimé pouvoir se tourner vers les éléments d'architecture de Carhaix pour dater plus précisément l'exploitation. Un bloc de corniche modillonnaire, que nous proposons de dater de l'époque julio-claudienne, et deux chapiteaux toscans, que leur contexte conduit à attribuer au début du IV s. , nous offrent les deux termes d'un arc chronologique qui couvre presque tout le Haut-Empire. Les blocs conservés sont malheureusement trop peu nombreux pour nous permettre de distinguer les temps forts d'une activité sans doute discontinue, qui ne faisait que répondre à la demande des différents programmes architecturaux. On doit donc attendre beaucoup des fouilles récemment entreprises. Un sondage d'évaluation a été pratiqué en juillet 2000, à la périphérie nord-ouest de la carrière, à $17 \mathrm{~m}$ d'une source et à $35 \mathrm{~m}$ du front de taille le plus proche. Cette fouille d'étendue réduite

88. Une communication de M. Pichon au colloque d'Argenton-surCreuse a signalé l'utilisation d'un outil de même morphologie dans la carrière du Pont de Tendu, ouverte sur la rive gauche de la Bouzanne dans la première moitié du I ${ }^{\mathrm{cr}} \mathrm{s}$. I ce texte publié dans les actes du colloque (Lorenz ot al., 2000, p. 25) n'aborde pas cette question.

89. I'église de Locuon constitue le plus ancien témoin d'une exploitation de la carrière à l'époque moderne. Pour sa datation, voir Danigo, 1996, p. 72. 
(11 m x $3 \mathrm{~m}$ ) a révélé une stratigraphie complexe : à la base, reposant sur le paléosol, une couche d'occupation a livré du mobilier céramique et des blocs travaillés, correspondant à une première phase de l'activité. Ce niveau a été recouvert d'un remblai constitué de déchets de carrière, grosses pierres brutes provenant vraisemblablement de la découverte, mais aussi éclats plus réduits et sable résultant de l'emploi de l'escoude*. Sur ce sol nivelé et assaini, a été bâti un édifice en petit appareil soigné, dont on a retrouvé la base de deux murs. La proximité de la fontaine, ainsi que l'abondance relative du mobilier céramique recueilli donnent à penser que l'on est ici en présence d'un habitat de carriers. Cet édifice a ensuite lui-même été recouvert par des déchets de carrière. Dans un dernier stade, une forge a probablement été installée à proximité immédiate: aucune structure n'a encore été reconnue, mais le site est recouvert d'une épaisse couche noire très dense, comportant de nombreux déchets métalliques. Si les différentes phases n'ont pu être datées avec précision, le mobilier recueilli se situe entre le début du ${ }^{\mathrm{er}} \mathrm{s}$. et le milieu du II's.

$$
* *
$$

Les techniques d'extraction mises en ceuvre à Locuon témoignent d'une parfaite connaissance du matériau de la part de carriers qui sont à même de tirer le meilleur parti de la structure du massif. La méthode des havages* est certes très fastidieuse et coûteuse en temps; elle autorise en revanche l'application d'un canevas d'extraction très strict et permet un gain de temps sensible au moment de l'équarrissage des blocs.

Sans qu'il soit possible de proposer des indices de dureté précis, il est indubitable que le granite de Locuon occupe une place singulière dans la gamme des granites régionaux ; pour la grande majorité de ces derniers, la seule technique d'extraction envisageable est celle qui fait uniquement appel aux coins. Il est à cet égard tout à fait remarquable que l'utilisation de l'escoude n'ait jamais été relevée dans aucune carrière régionale, et ce, quelle que soit la période considérée.

Il convient donc de se demander comment ces techniques étrangères aux pratiques régionales ont été introduites dans la cité des Osismes. On ne peut malheureusement attendre aucun élément de réponse du mobilier exclusivement céramique mis au jour lors des fouilles récentes. L'hypothèse la plus plausible consiste à

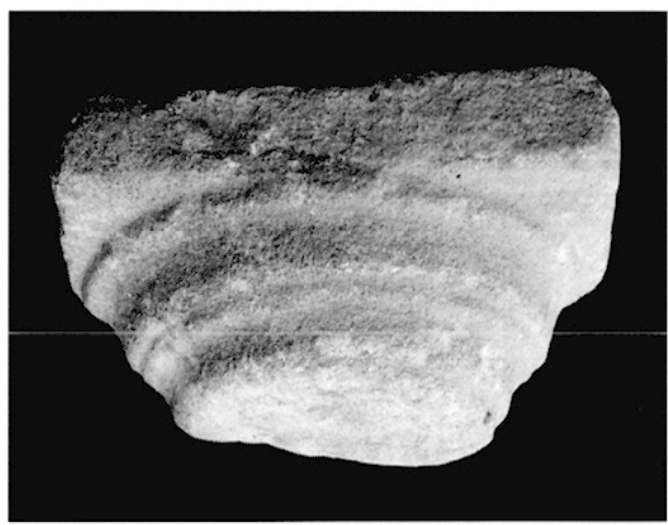

Fig. 109 - Chapiteau toscan tourné découvert au Centre hospitalier de Carhaix (photo Y. Maligorne, univ. de Brest).

envisager la venue, à l'occasion peut-être des premiers programmes monumentaux de Carhaix, de carriers spécialisés dans l'extraction du calcaire.

\section{DIFFUSION DE LA PRODUCTION}

Après examen de l'ensemble des éléments lapidaires romains connus dans la péninsule, l'aire de diffusion de la pierre de Locuon apparaît relativement restreinte : les environs immédiats de la carrière - encore que les témoins y soient peu nombreux - et Carhaix ont livré l'essentiel des indices.

Dans la capitale des Osismes, la majeure partie du décor architectonique est taillée dans le granite de Locuon. Ont été exhumés des chapiteaux, tous toscans, dont deux exemplaires tardifs présentent des traces de tournage (fig. 109), des tambours de colonnes lisses, un bloc de corniche modillonnaire. On signalera surtout les nombreux fragments d'un pilier, monument religieux ou funéraire (fig. 110). Datables du II's., ils sont abondamment ornés de rais-de-cœur, billettes, languettes, flots et oves; l'un des registres du monument portait en outre un décor figuré sculpté en haut relief, dont ne subsistent plus que de maigres vestiges. Ces éléments, qui n'ont pour l'heure aucun équivalent en Armorique, ne font que souligner la très bonne aptitude du granite de Locuon à supporter une taille fine.

D'autres granites ont été reconnus à Carhaix, qui proviennent tous du massif de Langonnet. Les volumes concernés sont si faibles que ces roches apparaissent plus comme des matériaux d'appoint que comme de réels concurrents. 


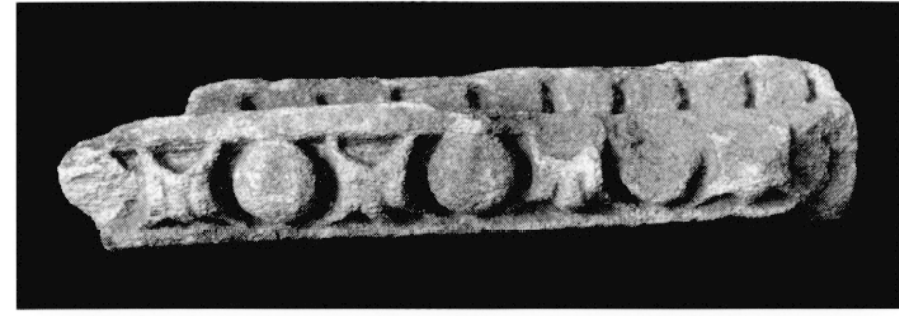

a

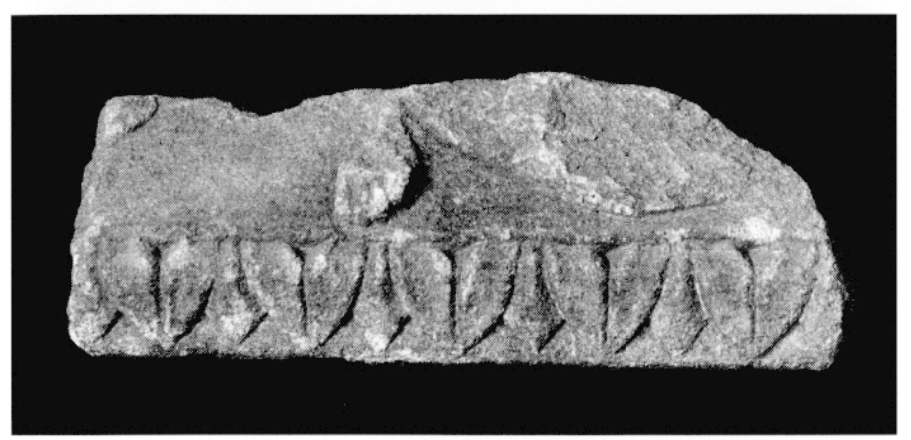

b

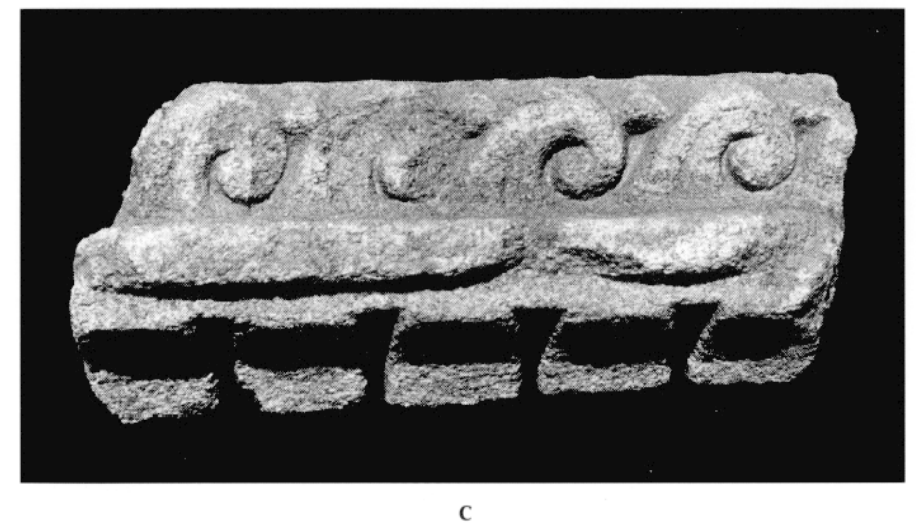

Fig. 110 - Fragments d'un pilier découverts rue de Kerniguez ì Carhaix (pholo J.-Y. Eveillard, univ. de Brest).

Hors de la capitale des Osismes, le granite de Locuon n'a pu être identifié qu'à Landeleau, à 13 km à l'ouest, où est conservé un imposant bloc d'architrave retaillé en sarcophage (fig. 111). Au-delà, d'autres massifs se posent en concurrents et les problèmes liés au transport, dans une région où les cours d'eau navigables sont rares, ont empêché une large diffusion du granite de Locuon.

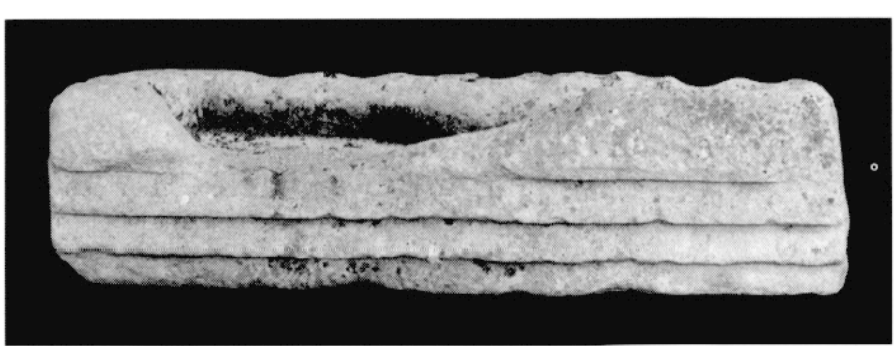

Fig. 111 - Bloc d'architrave réutilisé en sarcophage à Landeleau, Finistère, longueur : 2,42 m (photo J.-Y. Eveillard, univ. de Brest).

La question de l'acheminement des matériaux vers les chantiers de Vorgium/Carhaix peut être appréhendée avec une certaine vraisemblance dans la mesure où, comme on l'a dit, la carrière était située sur le passage d'une importante voie romaine. De l'altitude de $260 \mathrm{~m}$ au point de départ, on passe à celle de $150 \mathrm{~m}$ en l'espace de $27 \mathrm{~km}$. Deux vallées devaient être franchies, mais ni l'une ni l'autre ne constituait une difficulté insurmontable. Tout au plus peut-on penser que pour la plus importante, où l'on descend de la cote 160 à la cote 110 en l'espace de $1 \mathrm{~km}$, on devait faire preuve du maximum de précautions en attelant les bœufs à l'arrière. En nous appuyant sur les données fournies par J.C. Bessac pour les carrières du Bois des Lens, près de Nîmes (Bessac, 1996, p. 75-76 et supra, p. 29-51), nous avons calculé qu'un chargement de 4 à 6 t, tiré sur un chariot par deux ou trois paires de bœufs, pouvait atteindre Vorgium après $13 \mathrm{~h}$ de route. La difficulté a été singulièrement accrue lorsqu'il s'est agi de transporter des blocs d'architrave, évalués à 2,70 t, vers le site de Landeleau. Le trajet total est alors de $40 \mathrm{~km}$, avec le franchissement de deux vallées supplémentaires. Il aurait certainement été beaucoup plus facile de faire venir du granite du massif du Huelgoat, qui n'est éloigné que de $13 \mathrm{~km}$ en direction du nord, ou des environs de Le Saint, à $20 \mathrm{~km}$ vers le sud-est, deux gisements dont nous savons qu'ils étaient exploités à l'époque romaine. L'élection dans le cas présent de la pierre de Locuon traduit de manière éclatante la bonne réputation dont elle jouissait. 